\title{
Cardiac resident macrophages are involved in hypoxia-induced postnatal cardiomyocyte proliferation
}

\author{
BO LIU $^{1,2^{*}}$, HUA-GANG ZHANG ${ }^{1 *}$, YUN ZHU ${ }^{1}$, YUN-HAN JIANG ${ }^{1}$, \\ GUI-PING LUO $^{1}$, FU-QIN TANG ${ }^{1}$, ZHAO JIAN $^{1}$ and YING-BIN XIAO ${ }^{1}$ \\ ${ }^{1}$ Institute of Cardiovascular Surgery, Xinqiao Hospital, Third Military Medical University, \\ Chongqing 400037; ${ }^{2}$ Department of Cardiothoracic Surgery, The First Affiliated Hospital of \\ General Hospital of The Chinese People's Liberation Army, Beijing 100000, P.R. China
}

Received March 1, 2016; Accepted February 9, 2017

DOI: $10.3892 / \mathrm{mmr} .2017 .6432$

\begin{abstract}
Induction of cardiomyocyte proliferation, the most promising approach to reverse myocardial attrition, has been gaining importance as a therapy for cardiovascular disease. Hypoxia and macrophages were previously independently reported to promote cardiomyocyte proliferation in mice. However, whether hypoxia promotes cardiomyocyte proliferation in humans, and the association between hypoxia and macrophages in cardiomyocyte proliferation, have not to the best of our knowledge been previously investigated. The present study investigated the cardiomyocyte proliferation in 22 acyanotic and 29 cyanotic patients. Cardiomyocyte proliferation in a hypoxic mouse model $\left(15 \% \mathrm{O}_{2}\right)$ was subsequently performed and the macrophage subsets were analyzed. A C-C chemokine receptor type 2 (CCR2) inhibitor was used to increase the number of resident macrophages in order to investigate the effect of macrophages on cardiomyocyte proliferation. The results demonstrated that cardiomyocyte proliferation in the cyanotic infant group was significantly increased compared with the acyanotic infant group and the hypoxia-treated C57BL/6J neonates confirmed the hypoxia-induced cardiomyocyte proliferation. However, hypoxia did not induce the proliferation of isolated cardiomyocytes. Notably, hypoxia treatment increased the number of cardiac resident macrophages in neonate hearts. Furthermore, increasing the number of resident macrophages significantly enhanced cardiomyocyte proliferation. In conclusion, postnatal hypoxia promoted
\end{abstract}

Correspondence to: Professor Ying-Bin Xiao or Professor Zhao Jian, Institute of Cardiovascular Surgery, Xinqiao Hospital, Third Military Medical University, 183 Xinqiao Street, Chongqing 400037, P.R. China

E-mail: xiaoyb@tmmu.edu.cn

E-mail: zjian918@yahoo.com

*Contributed equally

Key words: hypoxia, resident macrophages, cardiomyocyte proliferation cardiomyocyte proliferation in humans and animals, and cardiac resident macrophages may be involved in this process. Therefore, this novel mechanism may provide a promising strategy for cardiovascular disease treatment.

\section{Introduction}

The inability to restore the damaged myocardium causes the major pathologies of cardiovascular disease. Therefore, induction of cardiomyocyte proliferation is a promising approach to reverse myocardial attrition, which may be applied to heart failure therapy $(1,2)$. Previous studies have confirmed that there is limited proliferative potential in the adult mammalian heart $(3,4)$. In the adult mouse heart, $\sim 10 \%$ of cardiomyocytes are mononuclear, of which $\sim 0.3 \%$ were induced to restart cytokinesis by the Neuregulin 1 signaling pathway (5). Previous studies have also provided certain information about cardiomyocyte renewal in humans, which indicated a rate of $1 \%$ at the age of 25 and $0.45 \%$ at the age of $75(6,7)$. Although the information gained about cardiomyocyte renewal in the adult mammal heart provided promise for cardiomyocyte proliferation, information regarding the detailed mechanisms of the modulation of cardiomyocyte proliferation is limited.

A previous study has confirmed the capacity for restoration in neonatal mouse hearts (8) and advanced research demonstrated that hypoxia promoted this regenerative process (9). The research suggested that hypoxia reduces oxidative stress, which relieves the DNA damage response, and the transition between oxygen levels in the embryonic and postnatal circulation was considered to be an important reason for cell-cycle arrest of mammalian cardiomyocytes soon after birth (9). This explanation is in accordance with the phenomenon of cardiac regeneration that occurs in urodele amphibians and zebrafish, which have an internal environment that is sustained at a lower oxygen state compared with mammals $(10,11)$. However, the phenomenon of hypoxia-induced cardiac proliferation remains unconfirmed in human hearts and the regulation of hypoxia-induced cardiac proliferation is not fully understood.

Macrophages are versatile immune cells that have a critical role in angiogenesis, organ development and injured tissue repair (12). Multiple reports have indicated an association between the immune system and regeneration in lower 
vertebrates, and supported a hypothesis that the mammalian immune system may influence the proliferative capacity. Specifically, macrophage infiltration was essential for limb regeneration in newts (13), indicating that alterations in macrophages represents a potential mechanism for the induction of tissue proliferation. Notably, a recent study confirmed that macrophages were essential for neonatal cardiomyocyte proliferation through promotion of myocardial angiogenesis (14). Further research indicated that distinct macrophage lineages had opposite effects on the restoration of the injured myocardium and the macrophages that promoted cardiac restoration are primarily composed of cardiac resident macrophages $(15,16)$. Although there are numerous existing associations between macrophages and cardiac restoration, whether macrophages are involved in hypoxia-induced cardiomyocyte proliferation has not previously been determined. The present study confirmed that cardiomyocyte proliferation occurred in human infant hearts, which was more significant in infants that suffered from chronic hypoxia. Furthermore, it was demonstrated that hypoxia increased the proportion of cardiac resident macrophages and this further contributed to postnatal cardiac proliferation.

\section{Materials and methods}

Patient samples. The present study was performed according to the principles outlined in the Declaration of Helsinki, informed consent was obtained from all subjects according to the procedures. The study was approved by the Human Ethics Committee of Xinqiao Hospital (Chongqing, China). The biopsy was taken from the stenotic right ventricular outflow tract and stored at $-80^{\circ} \mathrm{C}$ immediately. A total of 22 acyanotic and 29 cyanotic patients who had undergone cardiac surgery in Xinqiao Hospital (Chongqing, China) from June 2014-October 2015 were included. Patients were divided into 3 subgroups based on their age when surgery was performed, clinical characteristics are presented in Table I.

Animal breeding. C57BL/6J mice were bred in the animal facility of Xinqiao hospital. The investigation conformed to the Guide for the Care and Use of Laboratory Animals (17). All experiments were approved by the Animal Welfare Committee of Xinqiao Hospital. A total of 110 1-day-old mice were divided randomly into a normoxia group $(n=30)$, hypoxia group $(n=30)$, normoxia vehicle group $(n=25)$ and normoxia CCR2 inhibitor group $(n=25)$. The mice were fed by the mother at room temperature with free access to food and water and kept the rhythm of a $12 \mathrm{~h}$ light/dark cycle. The age- and sex-matched mice were sacrificed at differing times from 1 to 14 days following birth as described below. 5 mice per group were used for each experiment; $n=5$.

Hypoxia treatment. Hypoxia treatment was performed using a hypoxia workstation (Baker Ruskinn InvivO ${ }_{2}$ 1000; Ruskinn Technology, Ltd., Bridgend, UK) to maintain the environment at $15 \% \mathrm{O}_{2}$ content and $23^{\circ} \mathrm{C}$. The control group was treated in the same system but with $21 \% \mathrm{O}_{2}$ content.

Drug injection. A C-C chemokine receptor type 2 (CCR2) inhibitor (RS504393; Abcam, Cambridge, UK) was reconstituted in dimethyl sulfoxide to concentrations of $2.7 \mathrm{mg} / \mathrm{ml}$ for storage and diluted in PBS prior to use. Mice were weighed daily and $4 \mathrm{mg} / \mathrm{kg}$ CCR2 inhibitor was subcutaneously injected daily from days 0-10.

Immunostaining. Frozen human and mouse tissues were embedded in optimal cutting temperature (OCT) compound (tissue-tek 4583; Sakura Finetek USA Inc., USA) and $10 \mu \mathrm{m}$ cryosections were cut. Following fixation with $4 \%$ paraformaldehyde for $30 \mathrm{~min}$ at room temperature, sections were blocked with 10\% goat serum (AR0009; Boster Systems, Inc., Pleasanton, CA, USA) for $1 \mathrm{~h}$ at $37^{\circ} \mathrm{C}$ and incubated with primary antibodies overnight at $4^{\circ} \mathrm{C}$. Subsequently, sections were washed with PBS and incubated with secondary antibodies for $1 \mathrm{~h}$ at room temperature. The following primary antibodies were used: Anti-cardiac troponin T (TnT; cat. no. ab8295; 1:400; Abcam); anti-phospho histone H3 Ser10 (pH3; cat. no. 06-570; 1:200; EMD Millipore, Billerica, MA, USA); anti-Aurora B (cat. no. ab2254; 1:200; Abcam); and anti-wheat germ agglutinin (WGA) conjugated to Alexa Fluor 488 (cat. no. W11261; 1:200; Invitrogen; Thermo Fisher Scientific, Inc.). The following secondary antibodies were used: Alexa Fluor 488-labeled Goat Anti-Rabbit IgG (cat. no. A0423; 1:400; Beyotime Institute of Biotechnology, Haimen, China) and Alexa Fluor 647-labeled Goat Anti-Mouse IgG (cat. no. A0473; 1:400; Beyotime Institute of Biotechnology, Haimen, China). Immunofluorescence was visualized on a Leica confocal microscopy system (Leica Microsystems, Inc., Buffalo Grove, IL, USA) with the function of z-tack which revealed the vertical plane images of tissue sections. DAPI was used to stain nuclei. Results were quantified by examining $\geq 3$ similarly oriented sections from 3 independent samples in a blinded manner using Image J 2.1 software (National Institutes of Health, Bethesda, MD, USA).

Cell isolation. Mice were anesthetized with isoflurane and decapitated. Hearts were perfused with cold PBS, finely minced and digested with $1 \mathrm{mg} / \mathrm{ml}$ collagenase II (Sigma-Aldrich; Merck KGaA, Darmstadt, Germany) and $0.15 \mathrm{mg} / \mathrm{ml}$ DNase I (cat. no. 10104159001; Roche Diagnostics $\mathrm{GmbH}$, Mannheim, Germany) for $30 \mathrm{~min}$ at $37^{\circ} \mathrm{C}$ during constant agitation. The digested material was filtered through $40 \mathrm{~mm}$ filters and centrifuged at $4^{\circ} \mathrm{C}$ in $400 \times \mathrm{g}$ for $5 \mathrm{~min}$ in Dulbecco's modified Eagle's medium (Gibco; Thermo Fisher Scientific, Inc., Waltham, MA, USA) with $2 \%$ fetal calf serum (Gibco; Thermo Fisher Scientific, Inc., Waltham, MA, USA). Red blood cells were lysed in ammonium-chloride-potassium lysis buffer (Tiangen Biotech Co., Ltd., Beijing, China) and resuspended in fluorescence-activated cell sorting buffer (FACS; PBS containing 2\% FCS and 2 mM EDTA), as previously described (13).

Flow cytometry. Cell suspensions $\left(1 \times 10^{7}\right.$ cells in $\left.100 \mu \mathrm{l}\right)$ were incubated with Fc Block (cat. no. 101319; 1:100; BioLegend, Inc., San Diego, CA, USA) at $4^{\circ} \mathrm{C}$ for $5 \mathrm{~min}$ and labeled with the following fluorescently conjugated antibodies: Anti-CD45 APC (cat. no. 103111; 1:100) anti-Ly-6 G PerCP/Cy5.5 (cat. no. 127165; 1:100); anti-F4/80 PE/Cy7 (cat. no. 123113; 1:100); anti-MHC-II FITC (cat. no. 116405; 1:100) all obtained from BioLegend, Inc. and anti-CCR2 PE (cat. no. FAB5538P; 
Table I. Characteristics of patients.

\begin{tabular}{|c|c|c|c|c|c|c|}
\hline & \multicolumn{2}{|c|}{ Infant } & \multicolumn{2}{|c|}{ Adolescent } & \multicolumn{2}{|c|}{ Adult } \\
\hline & Acyanotic & Cyanotic & Acyanotic & Cyanotic & Acyanotic & Cyanotic \\
\hline No. & 13 & 18 & 6 & 7 & 3 & 4 \\
\hline Age, years & $1.6(0.3-4.0)$ & $1.4(0.5-3.5)$ & $8.5(6.0-14.0)$ & $10.0(6.0-15.0)$ & $25.0(21.0-30.0)$ & $31.0(28.0-36.0)$ \\
\hline Gender, male/female & $8 / 5$ & $12 / 6$ & $4 / 2$ & $2 / 5$ & $1 / 2$ & $1 / 3$ \\
\hline Oxygen saturation, $\% \mathrm{n}$ & $\begin{array}{c}98.0 \\
(97-100)\end{array}$ & $\begin{array}{c}75.2 \\
(65.0-80.0)^{\mathrm{a}}\end{array}$ & $\begin{array}{c}99.0 \\
(98.0-100)\end{array}$ & $\begin{array}{c}76.0 \\
(70.0-80.0)^{\mathrm{a}}\end{array}$ & $\begin{array}{c}99.0 \\
(97.0-100)\end{array}$ & $\begin{array}{c}82.0 \\
(80.0-86.0)^{\mathrm{a}}\end{array}$ \\
\hline \multicolumn{7}{|l|}{ Pathology } \\
\hline TOF & & 15 & & 7 & & 4 \\
\hline VSD and PA & & 3 & & & & \\
\hline VSD & 6 & & & & & \\
\hline RVOS & & & 2 & & & \\
\hline VSD and RVOS & 7 & & 4 & & 3 & \\
\hline
\end{tabular}

Data are presented as the median (minimum-maximum) or number (\%) of observations. TOF, Tetralogy of Fallot; VSD, ventricular septal defect; PA, pulmonary atresia; RVOS, right ventricular outflow tract obstruction. ${ }^{\mathrm{a}} \mathrm{P}<0.05$ vs. acyanotic group.

R\&D systems, Minneapolis, MN, USA) for 30 min at $4^{\circ} \mathrm{C}$. Cells were washed twice in FACS buffer. Flow cytometry analysis was performed on a flow cytometer (BD FACSCanto II; BD Biosciences, Franklin Lakes, NJ, USA) and data analysis was performed using the FlowJo 10.0 software (Tree Star, Inc., Ashland, OR, USA).

Statistical analysis. Data were analyzed using GraphPad Prism 6.0 software (GraphPad Software, Inc., La Jolla, CA, USA) and are presented as the mean \pm standard error of the mean. Comparisons between groups were performed using an unpaired two-tailed Student's t-test. Each experiment was repeated 3 times. $\mathrm{P}<0.05$ was considered to indicate a statistically significant difference.

\section{Results}

Clinical characteristics of patients. A total of 22 acyanotic and 29 cyanotic patients were included in this study, patients were divided into the following subgroups based on their age at the time of the operation: Infant group; adolescent group; and adult group. Clinical data are presented in Table I. Patients in the cyanotic group primarily underwent operations for Tetralogy of Fallot, while patients that suffered from ventricular septal defect combined with right ventricular outflow tract stenosis functioned as controls. The 2 groups were matched for age. Oxygen saturation of arterial blood was the primary difference between the 2 groups.

Cardiomyocyte proliferation in cyanotic and acyanotic patients. To exclude the potential effects of pressure overload on cardiomyocyte proliferation, cardiac samples were taken from the highest point of right ventricular outflow tract obstruction (Fig. 1A) (18). Cell size quantification demonstrated no significant difference between cardiomyocyte cell size of infants with and without cyanosis (Fig. 1B). To investigate cardiomyocyte proliferation in infants with and without cyanosis, cardiomyocyte mitosis was investigated by immunostaining using anti-pH3, a specific marker of G2-M progression, while cardiomyocytes were marked by anti-cardiac TnT antibody. High-resolution confocal z-stacking microscopy, a gold standard method for identifying colocalizations, was used to confirm the colocalization of $\mathrm{pH} 3$ signal and cardiomyocyte nucleus (Fig. 1C, bottom left). Quantification of the cardiomyocytes with nuclear $\mathrm{pH} 3$ signal (Fig. 1C, bottom right) demonstrated that $\mathrm{pH} 3$-positive cardiomyocytes were $\sim 10$-fold higher in the cyanotic infant group compared with the acyanotic infant group, which indicated that infant cardiomyocytes had mitotic potential and hypoxia enhanced this capacity.

A previous report indicated that the loss of regenerative capacity of adult cardiomyocytes was due to an inability to undergo cytokinesis, which may explain why the majority of adult cardiomyocytes were reported to exhibit polyploidy (19). Therefore, the present study investigated the cytokinesis of cardiomyocytes to further investigate the proliferation of cardiomyocytes in vivo. The presence of the cytokinesis marker, Aurora B kinase, was quantified and a significant increase in Aurora B-positive cardiomyocytes was observed in cyanotic samples compared with acyanotic samples ( $\mathrm{P}<0.05$; Fig. 1D). Strict $\mathrm{z}$-stack imaging, without the use of 2-dimensional imaging, was also performed as Aurora B may localize in the cleavage furrow between 2 myocytes that are not necessarily in the same horizontal plane. This technique increases the sensitivity and specificity of identifying true cardiomyocyte cytokinesis. These results indicated that sustaining hypoxia in the human heart following birth may enhance cardiomyocyte proliferation.

Effect of hypoxia on cardiomyocyte proliferation. To demonstrate the association between hypoxia and cardiomyocyte proliferation, neonatal mice were exposed to a mild hypoxic $\left(15 \% \mathrm{O}_{2}\right.$ content) or normoxic $\left(21 \% \mathrm{O}_{2}\right.$ content $)$ environment at the time of birth for several days (Fig. 2A). Hypoxia was confirmed by oxygen saturation, as the mean value was 

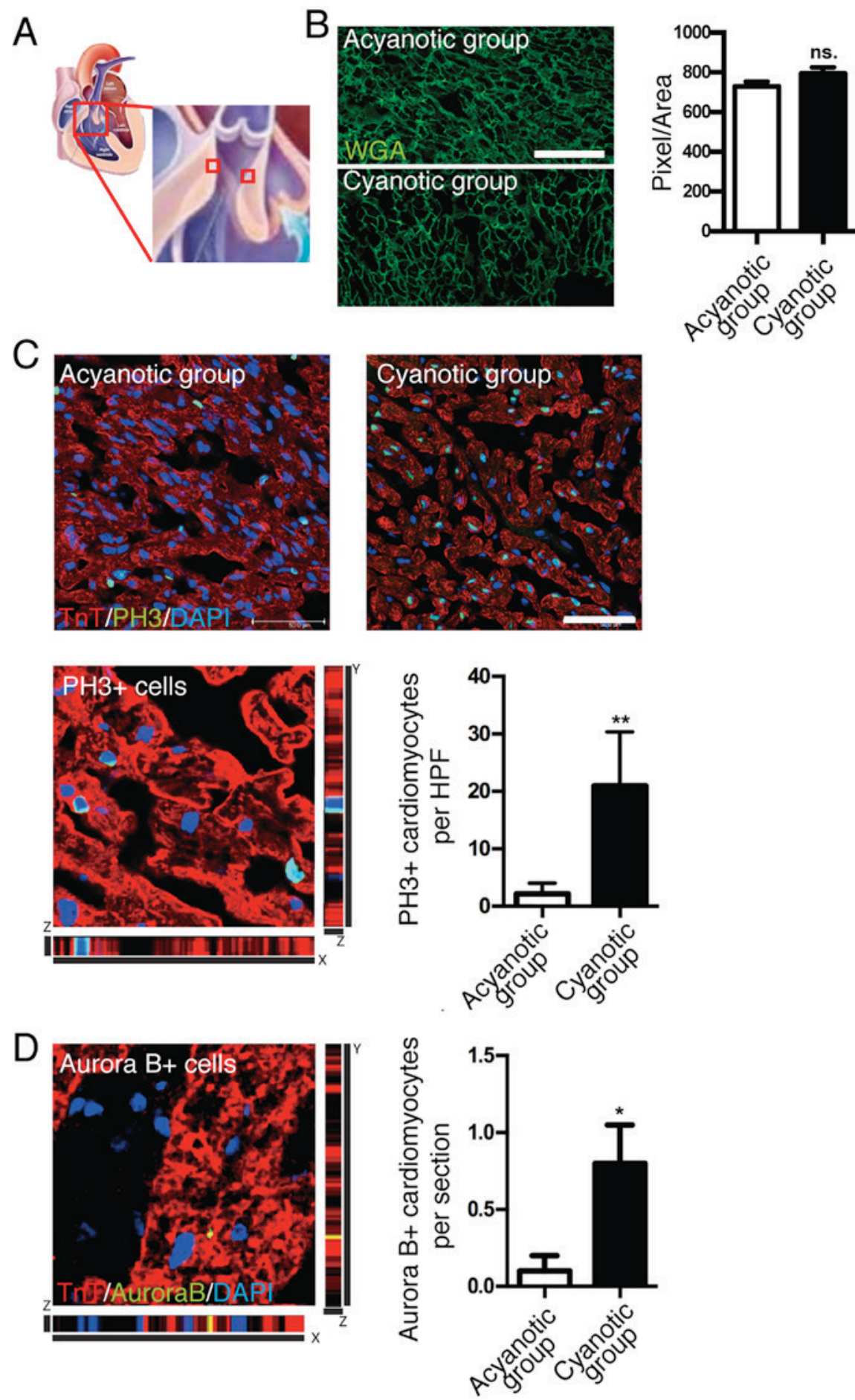

Figure 1. Effect of hypoxia on cardiomyocyte proliferation in human infants. (A) Myocardial tissues were taken from the distal obstructive right ventricular outflow tract. (B) Cardiomyocyte cell size was not significantly different between acyanotic and cyanotic infants; scale bar, $50 \mu \mathrm{m}$. (C) Coimmunostaining with $\mathrm{pH} 3$ and cardiac $\mathrm{TnT}$ antibodies demonstrated a significant increase in cardiomyocyte mitosis in the myocardium of cyanotic patients compared with acyanotic patients; scale bar, $50 \mu \mathrm{m}$. (D) Representative image of coimmunostaining with anti-Aurora B and cardiac TnT antibodies demonstrated increased cytokinesis in the myocardium of cyanotic patients. Data is presented as the mean + standard error of the mean. ${ }^{*} \mathrm{P}<0.05$ and ${ }^{* *} \mathrm{P}<0.01$ vs. acyanotic group. $\mathrm{PH} 3$, phospho histone H3 Ser10; TnT, troponin T; WGA, wheat germ agglutinin; HPF, high power field; P, days after birth; ns., not significant.

$\sim 85 \%$ in the hypoxic group. The heart weight to body weight $(\mathrm{HW} / \mathrm{BW})$ ratio was significantly increased in neonates under hypoxia compared with control mice $(\mathrm{P}<0.05$; Fig. 2B). Cardiomyocyte mitosis was measured by coimmunostaining with anti-pH3, anti-TnT antibody and DAPI (Fig. 2C), and results demonstrated that cardiomyocyte mitosis was significantly increased in the hypoxic group compared with control mice $(\mathrm{P}<0.01)$. In addition, localization of Aurora B kinase at the cleavage furrow (Fig. 2D) was significantly increased in the hypoxic group compared with control mice $(\mathrm{P}<0.05)$. The results indicate that a postnatal hypoxic state may directly enhance cardiomyocyte proliferation. To understand the regulative mechanism of cardiomyocyte proliferation in hypoxia, neonatal mouse cardiomyocytes were isolated and cultured in 
A

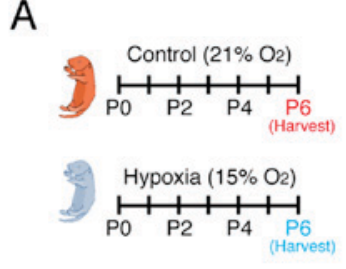

C
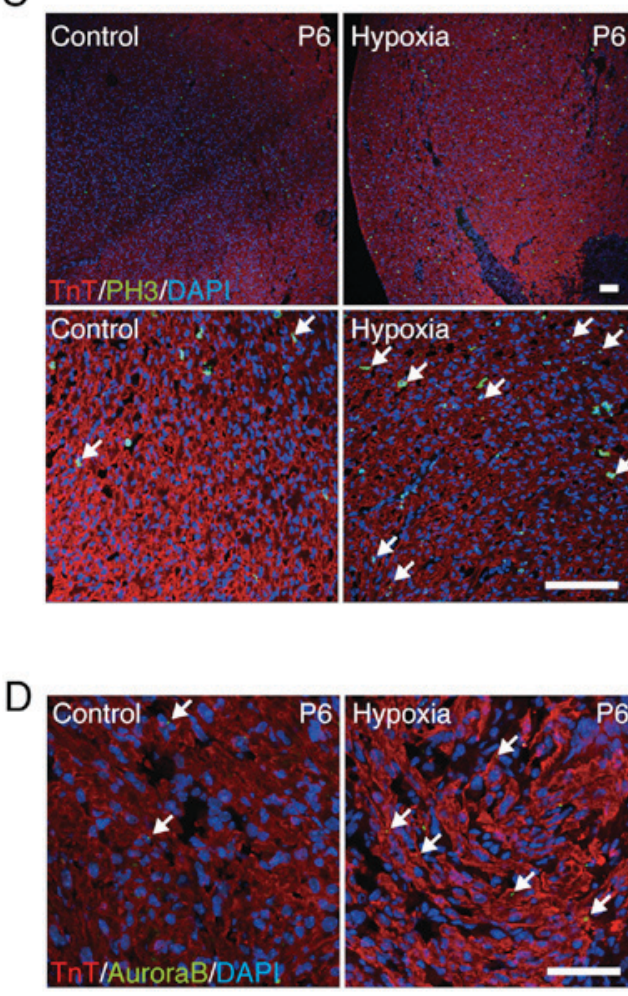

B

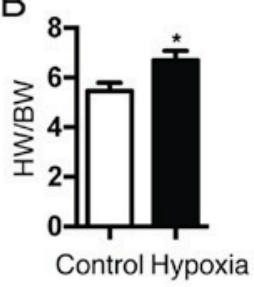

P6
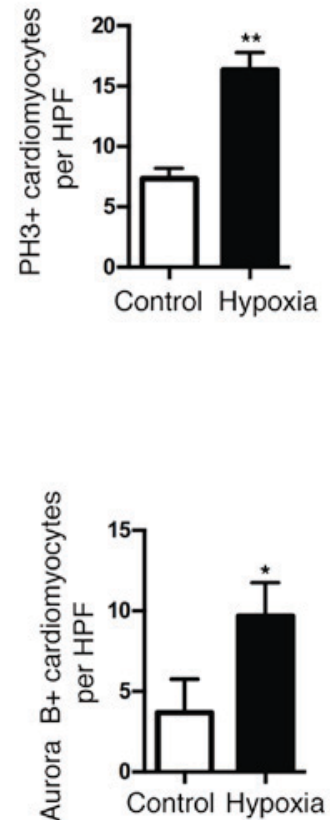

Figure 2. Effect of hypoxia on cardiomyocyte proliferation in neonatal mice. (A) Neonates were exposed to a normoxic or hypoxic environment at birth for 6 days. (B) HW/BW ratio was significantly increased in mice exposed to hypoxia. (C) Coimmunostaining demonstrated an increase in cardiomyocyte mitosis in hypoxia-exposed hearts; scale bar, $100 \mu \mathrm{m}$. (D) Coimmunostaining with anti-Aurora B and anti-TnT antibodies demonstrated increased cytokinesis in hypoxic hearts; scale bar, $50 \mu \mathrm{m}$. Data is presented as the mean + standard error of the mean. ${ }^{*} \mathrm{P}<0.05$ and ${ }^{* *} \mathrm{P}<0.01$ vs. control. HW/BW, heart weight/body weight; TnT, troponin T; P, days after birth; PH3, phospho histone H3 Ser10; HPF, high power field.

a hypoxic environment $\left(1 \% \mathrm{O}_{2}\right)$. The proliferation of cardiomyocytes at different time point was subsequently investigated. Unfortunately, after testing pH3 or Aurora B kinase-positive cardiomyocytes by immunostaining, no significant difference was observed between normoxic and hypoxic groups, which indicated that hypoxia alone may not promote the proliferation of isolated cardiomyocytes, and the microenvironment of cardiomyocytes may have a critical role in cardiomyocyte proliferation.

Effect of hypoxia on distinct cardiac macrophage subsets. A previous study indicated that macrophages were indispensable for neonatal heart regeneration (12). As distinct subsets of cardiac macrophages had the opposite effects (13), it was crucial to identify the subsets of macrophages that had a critical role in cardiomyocyte proliferation. Therefore, the present study examined the dynamics of cardiac macrophage subsets under sustained hypoxia following birth (Fig. 3A) and analyzed the macrophage subsets using the gating strategy as presented in Fig. 3B. The results demonstrated that the neonatal heart primarily contains 2 macrophage subsets; 1 resident macrophage (MHC-II $\left.{ }^{\text {low }} \mathrm{CCR} 2-\right)$ and 1 monocyte-derived immature macrophage (MHC-II ${ }^{\text {low }} \mathrm{CCR} 2+$ ). In response to hypoxia, the number of $\mathrm{MHC}-\mathrm{II}^{\text {low }} \mathrm{CCR} 2+$ monocyte-derived macrophages in the neonatal heart was reduced and the number of $\mathrm{MHC}-\mathrm{II}^{\text {low }} \mathrm{CCR} 2$-resident macrophages was increased (Fig. 3B). In addition, it was observed that hypoxia decreased monocyte-derived macrophages and increased resident macrophages in postnatal hearts along with the growth in a short time following birth, however, significant differences were not observed at postnatal days 10 or 14 (Fig. 3C). Notably, it was observed that the dynamic of distinct subsets is in accordance with the physiological rule of neonatal cardiac proliferation $(8,9)$. The results indicated that distinct subsets of cardiac macrophages may be responsible for the distinct effects on hypoxia-induced cardiomyocyte proliferation, however, the role of distinct macrophage subsets remains unclear. 
A
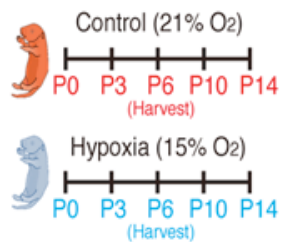

C

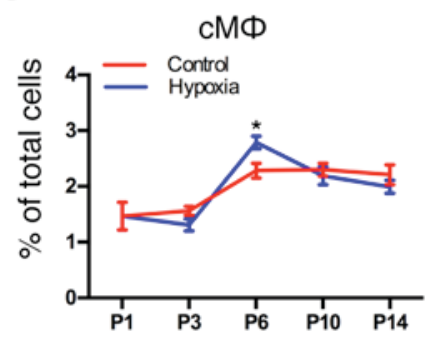

CCR2- cells
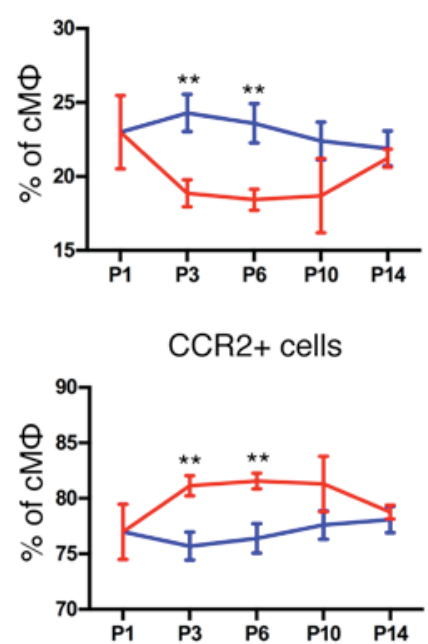

B

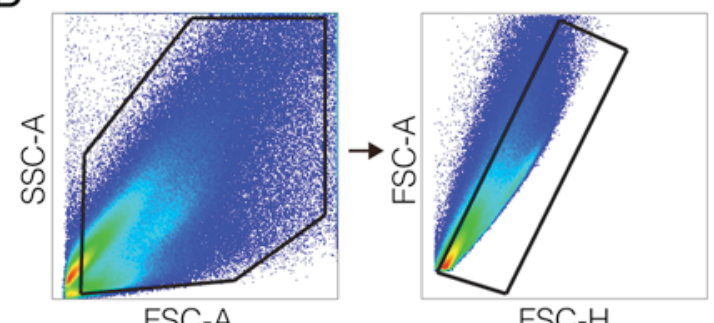

FSC-H

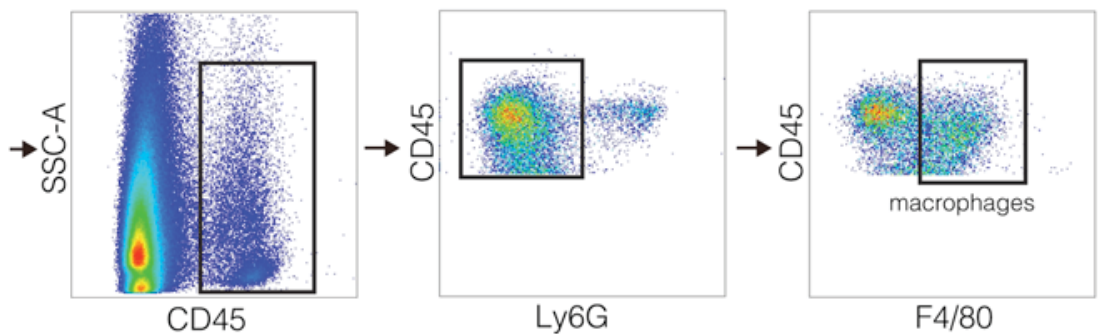

$\mathrm{F} 4 / 80$

$\square$ CCR2+ cells

CCR2- cells

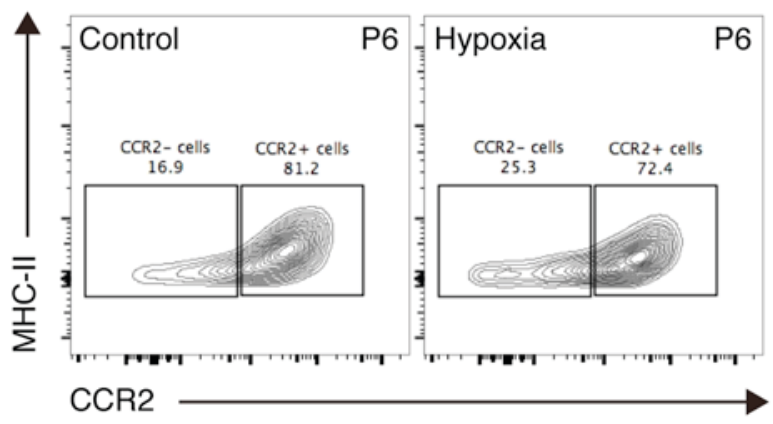

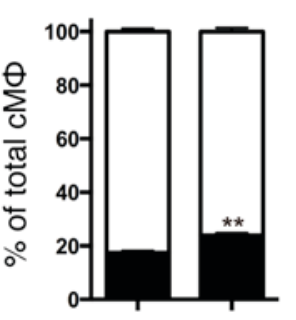

Control Hypoxia

Figure 3. Effect of hypoxia on distinct lineages of cardiac macrophages. (A) Neonates were exposed to a normoxic or hypoxic environment at birth for 2 weeks. (B) Gating strategy and representative fluorescence activated cell sorting analysis of macrophage populations in the neonatal heart on 6th day under normoxic and hypoxic conditions. (C) Quantification of macrophage populations in the neonatal heart in normoxic and hypoxic environments at various time points after birth. Data is presented as the mean \pm standard error of the mean. ${ }^{*} \mathrm{P}<0.05$ and ${ }^{* *} \mathrm{P}<0.01$ vs. control. P, days after birth; SSC, side scatter; FSC, forward scatter; Ly6G, lymphocyte antigen 6 complex locus G; CCR2, C-C chemokine receptor type 2; MHC II, histocompatibility-2 MHC; cM

Effect of cardiac resident macrophages on cardiomyocyte proliferation. To investigate whether the dynamics of distinct cardiac macrophage subsets contribute to cardiomyocyte proliferation in postnatal hearts, the recruitment of mononuclear phagocytes was inhibited by a selective CCR2 inhibitor (Fig. 4A). Although the absolute number of F4/80+ mononuclear phagocytes was only marginally affected, CCR2 inhibition resulted in significant alterations in resident macrophage subsets (Fig. 4B). CCR2 inhibition significantly reduced the recruitment of monocyte-derived macrophages into the neonatal heart and expanded the resident subsets (Fig. 4C). Colocalization of nuclear $\mathrm{pH} 3$ signal and cardiomyocytes indicated that cardiomyocyte mitosis was significantly increased in the CCR2 inhibition group compared with the vehicle group $(\mathrm{P}<0.01$; Fig. 4D). Cardiomyocyte cytokinesis was measured by localization of Aurora B kinase at the cleavage furrow, and the results demonstrated that Aurora B kinase-positive cardiomyocytes were significantly increased in the CCR2 inhibition group compared with the vehicle group $(\mathrm{P}<0.05$; Fig. 4E). These results demonstrated that the dynamics of distinct cardiac macrophage subsets influenced the proliferation of cardiomyocytes in postnatal hearts and resident macrophages served an essential role in the promotion of postnatal cardiomyocyte proliferation.

\section{Discussion}

The association between oxygen state and the proliferation of mammalian cardiomyocytes following birth has been previously demonstrated in animal models $(9,11)$, however, similar findings have not been reported in humans due to the difficulty of collecting appropriate samples. The present study collected myocardial samples from cyanotic congenital heart disease (CHD) patients, whose internal environment has a sustained hypoxic state since birth, which were compared with samples from acyanotic CHD patients. This is an appropriate study to 
A

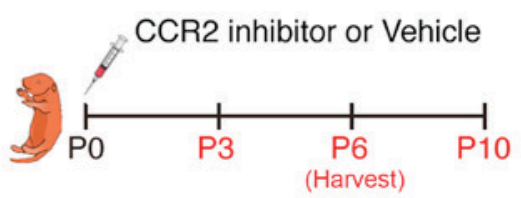

C

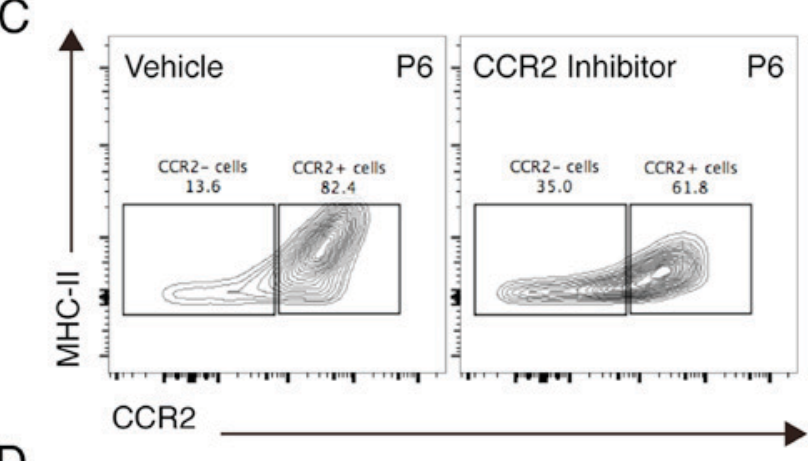

D

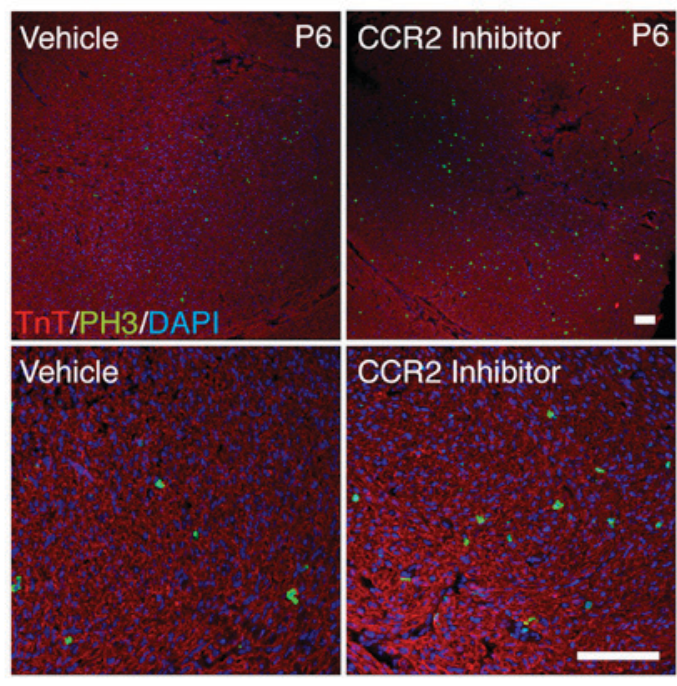

E

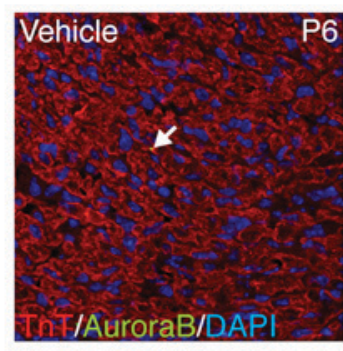

B

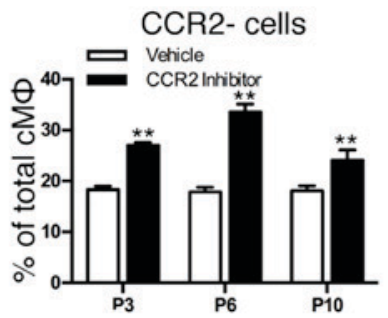

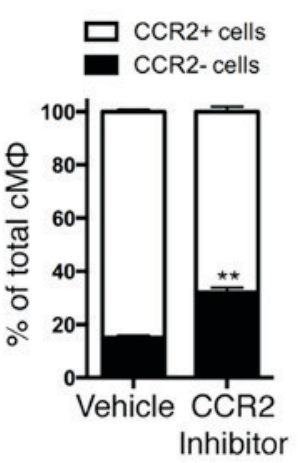
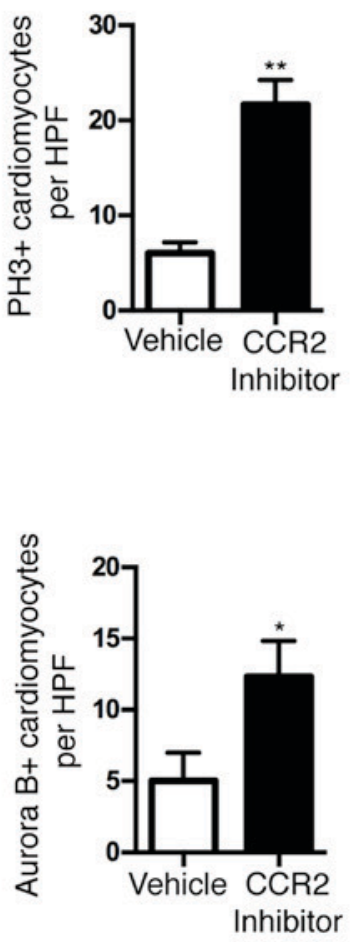

Figure 4. Effect of cardiac resident macrophages on postnatal cardiomyocyte proliferation. (A) CCR2 inhibitor (3 mg/kg, daily) was injected subcutaneously after birth for 2 weeks and hearts were harvested at different time points. (B) Quantification of cardiac macrophage populations. (C) Representative image of fluorescence activated cell sorting analysis of cardiac macrophage populations on the 6th day. (D) Co-immunostaining with anti-pH3 and anti-TnT antibodies demonstrated a significant increase in cardiomyocyte mitosis in the CCR2 inhibitor-treated subjects; scale bar, $100 \mu \mathrm{m}$. (E) Coimmunostaining with anti-Aurora B and anti-TnT antibodies demonstrated increased cytokinesis in the hearts of CCR2 inhibitor-treated subjects; scale bar, $50 \mu \mathrm{m}$. Data are presented at the mean \pm standard error of the mean. ${ }^{*} \mathrm{P}<0.05$ and ${ }^{* * *} \mathrm{P}<0.01$ vs. vehicle. CCR2, C-C chemokine receptor type 2; PH3, phospho histone H3 Ser10; TnT, troponin T; P, days after birth; MHC II, histocompatibility-2 MHC; cMØ, cardiac macrophages; HPF, high power field.

investigate whether a sustained hypoxic state has an effect on the promotion of cardiomyocyte proliferation following birth in humans. In addition, the present study also performed analysis on adolescent and adult heart tissues; however, no significant differences between the cyanotic and acyanotic groups were observed and results were not presented. Combined with the results demonstrated by the hypoxic mouse model, the current study demonstrated that hypoxia promoted cardiomyocyte proliferation following birth. The present study, to the best of our knowledge, was the first to demonstrate that hypoxia 
affected the composition of macrophages in postnatal hearts, which may further contribute to cardiomyocyte proliferation. Several previous studies have demonstrated that macrophages were associated with the angiogenesis that promoted the repair of the infarct region of the myocardium (1,12-15). However, there were no previous reports that indicated that macrophages may be involved in hypoxia-induced cardiomyocyte proliferation. We hypothesize that macrophage-associated angiogenesis may be important for the effects observed on cardiomyocyte proliferation. The results have revealed a novel mechanism for the association among hypoxia, macrophages and cardiomyocyte proliferation; however, the details of this phenomenon require further investigation.

The ability of cardiac embryonic-derived macrophages, also known as cardiac resident macrophages, to renew the population reduces with increasing age and they are gradually replaced by monocyte-derived macrophages (16). The present study demonstrated that increasing cardiac resident macrophages, or reducing monocyte-derived macrophages, promoted the proliferation of cardiomyocytes. Furthermore, with increasing age, the decrease in the number of resident macrophages and their replacement with monocyte-derived macrophages may be partially explain why cardiomyocytes exit the cell cycle soon after birth. However, to determine the reason for this in detail, the differences in the excreted factors between cardiac resident macrophages and monocyte-derived macrophages requires investigation.

Despite the importance of the results presented, the present study does not provide a clear understanding of the mechanism of cardiomyocyte proliferation caused by increased resident macrophages and decreased monocyte-derived macrophages. Other limitations also include the small sample size in humans. More importantly, although there is clear evidence of hypoxia-induced cardiomyocyte proliferation via altered composition of cardiac macrophages, it is unclear whether it leads to an increased contractile function in the long term.

\section{Acknowledgements}

The present study was supported by the National Natural Science Foundation of China (grant nos. 81370004, 81270228 and 81471408).

\section{References}

1. Batty JA, Lima JA Jr and Kunadian V: Direct cellular reprogramming for cardiac repair and regeneration. Eur J Heart Fail 18: 145-156, 2016.
2. Sinagra $G$ and Fabris E: Direct cellular reprogramming: The hopes and the hurdles. Eur J Heart Fail 18: 157-159, 2016.

3. Kühn B, del Monte F, Hajjar RJ, Chang YS, Lebeche D, Arab S and Keating MT: Periostin induces proliferation of differentiated cardiomyocytes and promotes cardiac repair. Nat Med 13: 962-969, 2007.

4. Canseco DC, Kimura W, Garg S, Mukherjee S, Bhattacharya S, Abdisalaam S, Das S, Asaithamby A, Mammen PP and Sadek HA: Human ventricular unloading induces cardiomyocyte proliferation. J Am Coll Cardiol 65: 892-900, 2015.

5. Bersell K, Arab S, Haring B and Kühn B: Neuregulin1/ErbB4 signaling induces cardiomyocyte proliferation and repair of heart injury. Cell 138: 257-270, 2009.

6. Bergmann O, Bhardwaj RD, Bernard S, Zdunek S, Barnabé-Heider F, Walsh S, Zupicich J, Alkass K, Buchholz BA, Druid $\mathrm{H}$, et al: Evidence for cardiomyocyte renewal in humans. Science 324: 98-102, 2009.

7. Mollova M, Bersell K, Walsh S, Savla J, Das LT, Park SY, Silberstein LE, Dos Remedios CG, Graham D, Colan S and Kühn B: Cardiomyocyte proliferation contributes to heart growth in young humans. Proc Natl Acad Sci USA 110: 1446-1451, 2013.

8. Porrello ER, Mahmoud AI, Simpson E, Hill JA, Richardson JA, Olson EN and Sadek HA: Transient regenerative potential of the neonatal mouse heart. Science 331: 1078-1080, 2011.

9. Puente BN, Kimura W, Muralidhar SA, Moon J, Amatruda JF, Phelps KL, Grinsfelder D, Rothermel BA, Chen R, Garcia JA, et al: The oxygen-rich postnatal environment induces cardiomyocyte cell-cycle arrest through DNA damage response. Cell 157: 565-579, 2014

10. Poss KD, Wilson LG and Keating MT: Heart regeneration in zebrafish. Science 298: 2188-2190, 2002.

11. Jopling C, Suñé G, Faucherre A, Fabregat C and Izpisua Belmonte JC: Hypoxia induces myocardial regeneration in zebrafish. Circulation 126: 3017-3027, 2012.

12. Wynn TA, Chawla A and Pollard JW: Macrophage biology in development, homeostasis and disease. Nature 496: 445-455, 2013.

13. Godwin JW, Pinto AR and Rosenthal NA: Macrophages are required for adult salamander limb regeneration. Proc Natl Acad Sci USA 110: 9415-9420, 2013.

14. Aurora AB, Porrello ER, Tan W, Mahmoud AI, Hill JA, Bassel-Duby R, Sadek HA and Olson EN: Macrophages are required for neonatal heart regeneration. J Clin Invest 124: 1382-1392, 2014

15. Lavine KJ, Epelman S, Uchida K, Weber KJ, Nichols CG, Schilling JD, Ornitz DM, Randolph GJ and Mann DL: Distinct macrophage lineages contribute to disparate patterns of cardiac recovery and remodeling in the neonatal and adult heart. Proc Natl Acad Sci USA 111: 16029-16034, 2014.

16. Molawi K, Wolf Y, Kandalla PK, Favret J, Hagemeyer N, Frenzel K, Pinto AR, Klapproth K, Henri S, Malissen B, et al: Progressive replacement of embryo-derived cardiac macrophages with age. J Exp Med 211: 2151-2158, 2014.

17. U.S. Department of Heatth and Human Services, Public Health Service, National Institutes of Health (NIH): Guide for the care and use of laboratory animals. NIH, Bethesda, MD, 1985.

18. Brickner ME, Hillis LD and Lange RA: Congenital heart disease in adults. N Engl J Med 342: 988-a, 2000.

19. Senyo SE, Steinhauser ML, Pizzimenti CL, Yang VK, Cai L, Wang M, Wu TD, Guerquin-Kern JL, Lechene CP and Lee RT: Mammalian heart renewal by pre-existing cardiomyocytes. Nature 493: 433-436, 2013. 